Matthieu Brejon De Lavergnée, Olivier Tort (Dir.),L'Union du Trône et de l'Autel ? Politique et religion sous la Restauration

Paris, Presses universitaires de Paris Sorbonne, 2012, 252 p.

\title{
Bertrand Goujon
}

\section{(2) OpenEdition \\ Journals}

Édition électronique

URL : http://journals.openedition.org/assr/25182

DOI : $10.4000 /$ assr.25182

ISSN : $1777-5825$

Éditeur

Éditions de l'EHESS

Édition imprimée

Date de publication : 30 décembre 2013

Pagination : 150

ISSN : 0335-5985

Référence électronique

Bertrand Goujon, « Matthieu Brejon De Lavergnée, Olivier Tort (Dir.),L'Union du Trône et de l'Autel ? Politique et religion sous la Restauration », Archives de sciences sociales des religions [En ligne], 164 । 2013, mis en ligne le 18 février 2014, consulté le 21 septembre 2020. URL : http:// journals.openedition.org/assr/25182; DOI : https://doi.org/10.4000/assr.25182

Ce document a été généré automatiquement le 21 septembre 2020

(C) Archives de sciences sociales des religions 


\section{Matthieu Brejon De Lavergnée,} Olivier Tort (Dir.),L'Union du Trône et de l'Autel ? Politique et religion sous la Restauration

Paris, Presses universitaires de Paris Sorbonne, 2012, 252 p.

Bertrand Goujon

\section{RÉFÉRENCE}

Matthieu Brejon De Lavergnée, Olivier Tort (Dir.),L'Union du Trône et de l'Autel?

Politique et religion sous la Restauration, Paris, Presses universitaires de Paris

Sorbonne, 2012, $252 \mathrm{p}$. 
1 Issues d'une journée d'étude organisée en septembre 2008 à Paris, les contributions réunies dans cet ouvrage témoignent $\mathrm{du}$ regain d'intérêt que suscite la Restauration dans les rangs d'une nouvelle génération d'historiens et réinterrogent la notion d' " union du trône et de l'autel», communément admise pour désigner les relations entre pouvoir religieux et pouvoir politique sous les règnes de Louis XVIII et Charles X. De fait, la diversité des terrains envisagés - qui, inhérente à la publication d'actes, ne facilite pas toujours la lisibilité de la perspective d'ensemble, en dépit de l'agencement thématique choisi par les directeurs de la publication - témoigne d'une ambiguïté desdites relations qui se lit tant en termes institutionnels, administratifs et financiers

Matthieu Brejon de Lavergnée \& Olivier Tort (dir.)

\section{L'union du Trône et de l'Autel ?}

Politique et religion sous la Restauration

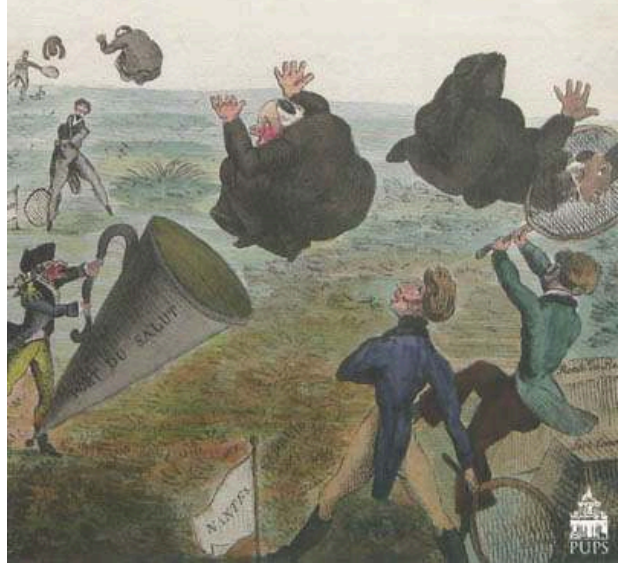
qu'idéologiques, mémoriels ou intellectuels.

2 La gestion publique des cultes dans le cadre concordataire révèle une politique religieuse du régime plus contrastée que ne le laisse supposer sa réputation de cléricalisme. Jean-Pierre Moisset souligne ainsi que si tous les gouvernements de la Restauration, sans distinction de ligne idéologique, contribuent à une augmentation du budget des cultes (+ $61 \%$ entre 1817 et 1829) qui profite à $98 \%$ à l'Église catholique, ils ont adopté une position budgétaire bienveillante à l'égard des pasteurs protestants : le contraste est à cet égard frappant avec le culte israélite, qui reste privé de la manne publique jusqu'à l'octroi des fonds nécessaires à l'ouverture de l'école rabbinique de Metz en 1829-1830, comme le rappelle Nadine Gastaldi, et n'est intégré de manière pérenne au budget de l'État que sous la monarchie de Juillet. De Richelieu à Polignac, la règle est au cofinancement des cultes avec les collectivités locales et la priorité est durablement donnée à la revalorisation des traitements des évêques plutôt qu'à celle des desservants, d'où la persistance d'une misère matérielle du clergé paroissial particulièrement sensible en milieu rural.

Celle-ci fournit aux députés et pairs ultras les arguments d'une «rhétorique de la pitié »(Olivier Tort) où la compassion le dispute à la dénonciation du danger social résultant de la paupérisation des prêtres et par laquelle les Chevaliers de la foi - qui n'en ont d'ailleurs pas le monopole - tentent d'obtenir l'instauration d'une dotation permanente pour l'Église. Encore cet objectif se heurte-t-il à l'opposition des libéraux, hostiles à toute idée d'une solidarité fiscale compensatoire et prompts à égratigner la vénalité épiscopale, ainsi qu'aux stratégies de contournement d'une partie de la droite, plus soucieuse d'économiser les deniers publics que d'appliquer le programme du "parti religieux». L'une des principales satisfactions de ce dernier réside dans la création en 1824 du ministère des Affaires ecclésiastiques confié à Mgr Frayssinous, dont Rémy Hême de Lacotte propose une triple réinterprétation. D’une part, les affaires 
religieuses sont ainsi émancipées de la tutelle du ministère de l'Intérieur. D'autre part, il s'agit de "faire rentrer l'Église dans l'État " - dans la même veine que la nomination de pairs ecclésiastiques qui viennent grossir les rangs des ultras et du centre droit à la Chambre haute entre 1822 et 1826, comme le signale Séverine Blenner-Michel en approfondissant les pistes déjà esquissées par Emmanuel de Waresquiel (Emmanuel de Waresquiel, Un groupe d'hommes considérables. Les pairs de France et la Chambre des pairs héréditaires de la Restauration, 1814-1831, Paris, Fayard, 2006). Enfin, le nouveau ministère est conçu comme un instrument de régulation au sein de l'Église de France dans le conflit juridictionnel qui oppose alors un épiscopat resté majoritairement gallican à la Grande aumônerie sensible aux thèses ultramontaines. Cette analyse permet ainsi de remettre judicieusement en perspective les démêlés de Jean-Marie de Lamennais avec la Grande aumônerie, sur lesquels l'édition récente des Mémoires du sarcastique et perspicace chanoine Le Sage - commentée par Samuel Gicquel - vient de jeter un jour nouveau (Samuel Gicquel [prés.], Mémoires Du Chanoine Le Sage. Le Diocèse De SaintBrieuc De La Fin De l'Ancien Régime A La Monarchie De Juillet, Rennes, PUR, 2012).

4 L'accent est également mis sur des aspects peu connus de la mobilisation du religieux dans les enjeux symboliques et mémoriels qui traversent le champ politique. Loin de se réduire à des offices ordinaires, les messes du Saint-Esprit qui sont rituellement célébrées la veille de l'ouverture des Chambres constituent des messes de souveraineté dont le cérémonial, scrupuleusement étudié par Thibaut Trétout à partir des archives de la Maison du roi, réaffirme la suprématie de la figure royale et les hiérarchies institutionnelles tout en reflétant - dans ses ajustements et accommodements - les défis que constituent pour le régime l'élection de l'abbé Grégoire ou la santé déclinante de Louis XVIII. Plus explicitement politique encore est l'implication de l'église dans l'orchestration de l'expiation du régicide lors des messes célébrées le 21 janvier à partir de 1816, qui s'inscrit dans le processus chaotique - et inachevé - de sacralisation de Louis XVI, érigé en roi martyr, et dont les accents contre-révolutionnaires sèment la discorde en province, vers laquelle Emmanuel Fureix étend ses investigations. Cette captation de la caution divine au profit de la légitimation des Bourbons restaurés a pour corollaire un déclin du phénomène des «faux dauphins »- lesquels mobilisent volontiers arguments mystiques et révélations prophétiques pour appuyer leurs prétentions - qu'analyse Paul Airiau et qui atteint symptomatiquement son étiage sous le règne de Charles $\mathrm{X}$.

5 Sont enfin mises en lumière des tentatives jusqu'à présent méconnues ou dénigrées dans l'historiographie de maintenir des positions religieuses et/ou confessionnelles face à une actualité qui les met potentiellement en péril. Pierre-Yves Kirschleger montre ainsi que dans les sermons prononcés par les pasteurs protestants au cours de la Première Restauration, l'interprétation providentialiste de la chute de l'Empire s'articule avec un respect péremptoire du principe d'autorité et une contribution à la surenchère expiatoire qui sont propres à consolider l'intégration nationale des confessions minoritaires ; bien qu'encore précaire et fragilisée par les incertitudes que nourrit le retour des Bourbons, celle-ci passe alors par une adhésion à la monarchie restaurée, obligeant à nuancer la légende historiographique de l'ancrage irréductible du protestantisme français à la gauche de l'échiquier politique. Quant à Matthieu Brejon de Lavergnée, il rétablit pleinement dans le paysage estudiantin parisien les réseaux catholiques et royalistes polarisés par la Société des Bonnes études: étroitement liée à la Congrégation dès sa fondation, particulièrement implantée à l'École de droit, celle-ci se veut le vecteur d'une culture de droite qui, d'emblée 
traversée par de profonds clivages religieux (entre tièdes et dévots, gallicans et ultramontains), résiste mal à la vague de contestation qui se lève dans ses rangs mêmes en 1827-1828.

6 Agrémenté d'un recueil d'illustrations hors texte qui fait la part belle aux caricatures anticléricales, cet ouvrage témoigne de la pertinence du prisme religieux pour saisir les tensions et les ambivalences qui ont traversé l'ensemble du champ politique sous la Restauration, comme le donnent à voir les terrains d'investigation multiples et complémentaires qui ont été envisagés. 